\title{
Smooth Numbers in Short Intervals
}

\author{
Ernie Croot * \\ School of Mathematics \\ Georgia Institute of Technology \\ Atlanta, Georgia 30332
}

June 6, 2006

\begin{abstract}
We show that for any $\epsilon>0$, there exists $c>0$, such that for all $x$ sufficiently large, there are $x^{1 / 2}(\log x)^{-\log 4-o(1)}$ integers $n \in$ $[x, x+c \sqrt{x}]$, all of whose prime factors are $\leq x^{47 /(190 \sqrt{e})+\epsilon}$.
\end{abstract}

\section{AMS Suject Classification. 11N25.}

Keywords. Smooth Numbers, Multiplicative Number Theory.

\section{Introduction.}

There are many unsettled questions concerning the distribution of integers having no "large" prime factors. Such integers are called "smooth numbers", and we say that an integer $n$ is $y$-smooth if all its prime divisors are $\leq y$. For an interval $[a, b]$ define $\psi([a, b], y)$ to be the number of $y$-smooths lying in $[a, b]$. The following is one such important, unsettled question:

\section{Conjecture 1}

$$
\psi\left([x, x+\sqrt{x}], x^{\theta}\right)>0, \text { for all } 0<\theta \leq 1 \text {, and all } x>x_{0}(\theta) .
$$

\footnotetext{
*Supported by NSF grant DMS-0500863
} 
Harman [6] showed that this conjecture holds for $y=x^{1 /(4 \sqrt{e})+\epsilon}$ and any $\epsilon>0$; moreover, he has shown that there are $\gg \sqrt{x}$ such $y$-smooths in $[x, x+\sqrt{x}]$, and has obtained results for even shorter intervals, at the expense of requiring larger values of $y$. For intervals of length much longer than $\sqrt{x}$, much more is known. For instance, Balog [1] showed that

$$
\psi\left(\left[x, x+x^{1 / 2+\epsilon}\right], x^{\alpha}\right)>x^{1 / 2+\epsilon-o_{\epsilon, \alpha}(1)},
$$

and many researchers have proved various refinements. For instance, Friedlander and Granville [3] showed that the interval width $x^{1 / 2+\epsilon}$ can be replaced with any

$$
z>y^{2} \sqrt{x} \exp \left((\log x)^{1 / 6}\right),
$$

and $x^{\alpha}$ with $y$, where

$$
\exp \left((\log x)^{5 / 6+o(1)}\right) \leq y \leq x .
$$

Friedlander and Lagarias [5] obtained results for intervals shorter than $x^{1 / 2}$, and showed that

$$
\psi\left(\left[x, x+x^{\beta}\right], x^{\alpha}\right) \gg_{\alpha, \beta} x^{\beta}, \text { whenever } \beta>1-\alpha-c \alpha(1-\alpha)^{3},
$$

where $c$ is an absolute positive constant. There are also some conditional improvements; for instance, Xuan [9] showed that the Riemann Hypothesis implies $\left[x, x+\sqrt{x}(\log x)^{1+o(1)}\right]$ contains an $x^{\epsilon}$-smooth.

In this paper we prove the following

Theorem 1 For every $\epsilon>0$, there exist $c=c(\epsilon)$, such that for all $x$ sufficiently large,

$$
\psi\left([x, x+c \sqrt{x}], x^{47 /(190 \sqrt{e})+\epsilon}\right)>\sqrt{x}(\log x)^{-\log 4-o(1)} .
$$

This result improves upon that of Harman's for intervals of length more than a constant times $\sqrt{x}$; however, Harman's still gives the strongest result for intervals of length exactly $\sqrt{x}$. It might be possible to refine the argument in our paper to beat Harman's result for intervals of length less than $\sqrt{x}$, but the argument would certainly be much more complicated. 


\section{Proof of Theorem 1.}

\subsection{Discussion of the Proof Strategy}

Let $\delta=\delta(\epsilon)$ be some parameter, which we will choose later. The proof will work by showing that a little more than half of the integers

$$
z \in Z:=\left[(1+\delta)^{-1} \sqrt{x},(1+\delta) \sqrt{x}\right] \cap \mathbb{Z}
$$

have the property that $[z, z+k]$ contains a $y=x^{47 / 190 \sqrt{e}+\epsilon}$-smooth integer. Here, $k=k(\epsilon)$ denotes a constant that depends only on $\epsilon$.

If we can show this, then, as we will explain in the next subsection, there must exists lots of pairs of integers

$$
z_{1} \in[(1-\delta) \sqrt{x}, \sqrt{x}], z_{2} \in[\sqrt{x},(1+\delta) \sqrt{x}], \text { with } z_{2}=\left\lceil x / z_{1}\right\rceil,
$$

such that both the intervals $\left[z_{1}, z_{1}+k\right]$ and $\left[z_{2}, z_{2}+k\right]$ contain $y$-smooth numbers $s_{1}$ and $s_{2}$, respectively. The product $s_{1} s_{2}$ is also $y$-smooth, and satisfies

$$
x \leq s_{1} s_{2}<x+3 k \sqrt{x} \text {. (for small enough } \delta>0 \text {.) }
$$

In fact, we will show that there are more than $\kappa \sqrt{x}$ such pairs $s_{1}, s_{2}$, where $\kappa=\kappa(\delta, \epsilon)>0$. This will prove our theorem in light of the following observation: Let $P$ denote the set of all these pairs, and note we have $|P|>$ $\kappa \sqrt{x}$. Among the $\left(s_{1}, s_{2}\right) \in P$, consider those satisfying

$$
\Omega\left(s_{1}\right), \Omega\left(s_{2}\right)<\log \log x+(\log \log x)^{2 / 3},
$$

where $\Omega(n)$ denotes the number of prime power divisors of $n$. As density $1-o(1)$ of the integers $n<2 \sqrt{x}$ satisfy this inequality, we conclude that $(1-o(1))|P|$ of our pairs $\left(s_{1}, s_{2}\right)$ do too. ${ }^{1}$ But then for each such pair we have

$$
\Omega\left(s_{1} s_{2}\right)<2 \log \log x+2(\log \log x)^{2 / 3}
$$

which implies

$$
\tau\left(s_{1} s_{2}\right)<(\log x)^{\log 4+o(1)} .
$$

\footnotetext{
${ }^{1}$ Note that no $s \in\left[(1+\delta)^{-1} \sqrt{x}, \sqrt{x}\right]$ appears more than $O(k)$ times as a first coordinate of a pair in $P$, and the analogous thing is true for the second coordinate. Thus, there are $\gg \sqrt{x}$ distinct first coordinates, and distinct second coordinates.
} 
Thus, the number of distinct products $s_{1} s_{2}$ such that $\left(s_{1}, s_{2}\right) \in P$ satisfies (1) is at least

$$
\frac{(1-o(1))|P|}{\max _{\left(s_{1}, s_{2}\right) \in P} \tau\left(s_{1} s_{2}\right)}>\sqrt{x}(\log x)^{-\log 4-o(1)},
$$

which would prove our theorem.

\subsection{Further Discussion}

Let $A$ denote the set of all integers $z \in Z$, such that

$$
\psi([z, z+k], y) \geq 1
$$

As we said before, if $|A|$ is a little more than $|Z| / 2 \sim \delta \sqrt{x}$, then we can make the above approach to proving our theorem work. More specifically, we will show that our argument will work if $x$ is sufficiently large and

$$
|A| \geq\left(\delta+\delta^{2}\right) \sqrt{x}
$$

Assume $|A|$ is indeed this large, and define the mapping

$$
\begin{aligned}
f: Z & \rightarrow Z \cup\{b\} \\
a & \rightarrow\lceil x / a\rceil,
\end{aligned}
$$

where $b$ is one more than the largest element of $Z$, and let $f_{A}$ denote the restriction of $f$ to $A \subseteq Z$.

Although $f$ is not injective, it almost is for small $\delta$ : First, define

$$
Z_{1}=\left[(1+\delta)^{-1} \sqrt{x}, \sqrt{x}\right) \cap \mathbb{Z} \text {, and } Z_{2}=[\sqrt{x},(1+\delta) \sqrt{x}] \cap \mathbb{Z},
$$

and note that $Z=Z_{1} \cup Z_{2}$. Now, $f$ maps $Z_{1}$ injectively into $Z_{2} \cup\{b\}$, and all but $O(1)$ integers in $Z_{1}$ are in the image of $f$ restricted to $Z_{2}$. Thus, for $x$ sufficiently large,

$$
|\operatorname{im}(f)| \geq 2\left|Z_{1}\right|+O(1) \geq\left(2 \delta-2 \delta^{2}\right) \sqrt{x}
$$

and therefore

$$
\left|\operatorname{im}\left(f_{A}\right)\right| \geq|A|-(|Z|+1-|\operatorname{im}(f)|)>\left(\delta-\delta^{3}\right) \sqrt{x} .
$$


This inequality follows since $f$ is at worst two-to-one (at least for $\delta$ small enough), and there are at most $|Z|+1-|\operatorname{im}(f)|$ points on $Z \cup\{b\}$ with two preimages in $Z$.

The fact that $f_{A}$ satisfies (3) implies

$$
\left|\operatorname{im}\left(f_{A}\right) \cap A\right| \geq|A|+\left|\operatorname{im}\left(f_{A}\right)\right|-|Z|>\left(2 \delta^{2}-2 \delta^{3}\right) \sqrt{x}
$$

Thus, there are more than $\left(2 \delta^{2}-2 \delta^{3}\right) \sqrt{x}$ pairs $(a,\lceil x / a\rceil) \in A \times A$, which is just the sort of conclusion we wanted.

\subsection{An Application of the Second Moment Method}

To show that $|A| \geq\left(\delta+\delta^{2}\right) \sqrt{x}$, and therefore complete the proof, we will apply the second moment method, along with a standard trick for reducing the smoothness bound (the standard trick is what contributes the factor $\sqrt{e}$ ).

Define

$$
D:=\left\{p_{1} p_{2}: p_{i} \text { prime }: p_{i} \in\left[x^{12 / 95-\epsilon / 3}, x^{12 / 95-\epsilon / 4}\right]\right\}
$$

and for an integer $n$, define

$$
h(n)=|\{q \in D: q \mid n\}| .
$$

The expected value of $h(n)$ over $n \in Z$ will be

$$
\mathbb{E}(h):=\frac{1}{|Z|} \sum_{n \in Z} h(n) \sim \sum_{q \in D} \frac{1}{q} .
$$

A lower bound for this expectation is

$$
\mathbb{E}(h) \gg \frac{1}{|Z|} \sum_{\substack{p_{1}, p_{2} \in\left[x^{12 / 95-\epsilon / 3}, x^{12 / 95-\epsilon / 4}\right] \\ p_{i} \text { prime }}} \frac{|Z|}{p_{1} p_{2}} \gg \epsilon^{2} .
$$


We will show that for fixed $\epsilon, \delta>0$ if $x>x_{0}(\delta, \epsilon)$ and $k>k_{0}(\delta, \epsilon)$, then

$$
\begin{aligned}
V & :=\sum_{z \in Z}\left(\sum_{n \in[z, z+k]} h(n)-(k+1) \mathbb{E}(h)\right)^{2} \\
& =\sum_{z \in Z}\left(\sum_{n \in[z, z+k]} h(n)\right)^{2}-(k+1)^{2} \mathbb{E}(h)^{2}|Z|+O(k) .
\end{aligned}
$$

is "small". What this will mean is that for "most" $z \in Z$ we will have

$$
\sum_{n \in[z, z+k]} h(n) \approx(k+1) \mathbb{E}(h) .
$$

At this point all we would get is that most of these intervals $[z, z+k]$ contain a number which is $x^{47 / 190+\epsilon}$ smooth, because if $h(n) \geq 1$ for some $n$ in this interval, then it factors as

$$
n=p_{1} p_{2} d, \text { where } p_{i} \geq x^{12 / 95-\epsilon / 3},
$$

which forces

$$
d<\frac{(1+\delta) x^{1 / 2}}{p_{1} p_{2}}<2 x^{47 / 190+2 \epsilon / 3}<x^{47 / 190+\epsilon} .
$$

(For $\delta<1$, of course.)

\subsection{Boosting the Smoothness}

To get that extra boost of $\sqrt{e}$ in the exponent of the smoothness bound, we show that "about half the time" the divisor $d$ above is $x^{47 / 190 \sqrt{e}+\epsilon}$ smooth. We begin by defining the modified weighting (a modification of $h(n)$ )

$$
h_{2}(n)=g(n) h(n), \text { where } g(n)= \begin{cases}1, & \text { if } n \text { is } x^{47 / 190 \sqrt{e}+\epsilon} \text { smooth; } \\ 0, & \text { otherwise. }\end{cases}
$$

Then, we have that

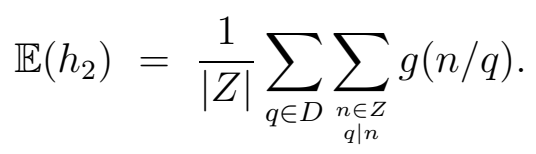


To estimate this inner sum we use the well-known fact that for $\epsilon_{0}>0$ there exists $\epsilon_{1}>0$ such that

$$
\psi\left(N, N^{1 / \sqrt{e}+\epsilon_{0}}\right) \sim\left(1 / 2+\epsilon_{1}\right) N .
$$

Thus, the inner sum in (6) exceeds $\left(1 / 2+\gamma_{1}\right)|Z| / q$, where $\gamma_{1}>0$ depends on $\epsilon>0$, and tends to 0 as $\epsilon$ tends to 0 ; and so,

$$
\mathbb{E}\left(h_{2}\right)>\left(1 / 2+\gamma_{1}\right) \mathbb{E}(h) .
$$

In the next subsection we will show that once

$$
0<\delta<\delta_{0}(\epsilon), k>k_{0}(\epsilon, \delta)
$$

and $x$ is sufficiently large, then

$$
V \leq \delta^{2} \mathbb{E}(h)^{2}(k+1)^{2}|Z| .
$$

It turns out that this implies that at least $\left(\delta+\delta^{2}\right) \sqrt{x}$ of the integers $z \in Z$ satisfy

$$
\psi([z, z+k], y)>0,
$$

which we know proves our theorem from comments in the previous subsection.

To see this last deduction, suppose that (9) holds and that, on the contrary, fewer than $\left(\delta+\delta^{2}\right) \sqrt{x}$ intervals $[z, z+k]$ satisfy (10). Let $Z^{\prime}$ denote the set of all $z \in Z$ such that $[z, z+k]$ is one of these intervals. We have

$$
\sum_{z \in Z}\left(\sum_{n \in[z, z+k]} h(n)\right)^{2} \geq \sum_{z \in Z^{\prime}}\left(\sum_{n \in[z, z+k]} h_{2}(n)\right)^{2}+\sum_{z \in Z \backslash Z^{\prime}}\left(\sum_{n \in[z, z+k]} h(n)\right)^{2} .
$$

To bound this first sum on the right-hand-side from below we note that all but $O(k)$ integers $n \in Z$ lie in exactly $k+1$ intervals $[z, z+k], z \in Z$; and so, by Cauchy-Schwarz, this quantity is at least

$$
\begin{aligned}
(k+1)^{2}\left|Z^{\prime}\right|^{-1} & \left(\sum_{n \in Z} h_{2}(n)\right)^{2}+O\left(k^{2}\right) \\
& =(k+1)^{2}\left|Z^{\prime}\right|^{-1}|Z|^{2} \mathbb{E}\left(h_{2}\right)^{2}+O\left(k^{2}\right) . \\
& \geq(k+1)^{2} \mathbb{E}\left(h_{2}\right)^{2}|Z|(2+O(\delta)) \\
& =(k+1)^{2} \mathbb{E}(h)|Z|\left(1 / 2+2 \gamma_{1}+2 \gamma_{1}^{2}+O(\delta)\right) .
\end{aligned}
$$


To bound the second sum on the right-hand-side of (11) from below, we first apply the triangle inequality $\|u\|_{2} \geq\|e\|_{2}-\|u-e\|_{2}$, where $u, e$ are vectors, with the coordinates of $u$ equal to $\sum_{n \in[z, z+k]} h(n)$ and the coordinates of $e$ equal to $(k+1) \mathbb{E}(h)$, and deduce

$$
\begin{aligned}
\left(\sum_{z \in Z \backslash Z^{\prime}}\left(\sum_{n \in[z, z+k]} h(n)\right)^{2}\right)^{1 / 2} & \geq(k+1) \mathbb{E}(h)\left|Z \backslash Z^{\prime}\right|^{1 / 2}-V^{1 / 2} \\
& \geq(k+1) \mathbb{E}(h)\left(\left|Z \backslash Z^{\prime}\right|^{1 / 2}-\delta|Z|^{1 / 2}\right) .
\end{aligned}
$$

Now, if $\delta>0$ is sufficiently small, then this last quantity is positive, and its square is easily seen to be at least

$$
\left.(k+1)^{2} \mathbb{E}(h)^{2}\left(\left|Z \backslash Z^{\prime}\right|-2 \delta|Z|\right)\right) \geq(k+1)^{2} \mathbb{E}(h)^{2}(1 / 2+O(\delta))|Z| .
$$

So, we deduce that

$$
\sum_{z \in Z}\left(\sum_{n \in[z, z+k]} h(n)\right)^{2} \geq(k+1)^{2}|Z| \mathbb{E}(h)^{2}\left(1+2 \gamma_{1}+2 \gamma_{1}^{2}+O(\delta)\right) .
$$

This and (5) implies that

$$
V \geq(k+1)^{2}|Z| \mathbb{E}(h)^{2}\left(2 \gamma_{1}+2 \gamma_{1}^{2}+O(\delta)\right),
$$

which contradicts (9) once $\delta>0$ is sufficiently small.

\subsection{Exponential Sums}

To prove (9), in light of (5) it suffices to obtain a very sharp upper bound for

$$
\begin{aligned}
\sum_{z \in Z}\left(\sum_{n \in[z, z+k]} h(n)\right)^{2} & =\left(\sum_{|j| \leq k}(k+1-|j|) \sum_{q_{1}, q_{2} \in D} \sum_{\substack{n \in Z \\
q_{1}\left|n, q_{2}\right| n+|j|}} 1\right)+O\left(k^{2}\right) \\
& =\left(2 \sum_{j=1}^{k}(k+1-j) \sum_{q_{1}, q_{2} \in D} \sum_{\substack{n \in Z \\
q_{1}\left|n, q_{2}\right| n+j}} 1\right)+O(k|Z|) .
\end{aligned}
$$


It is pretty clear, and easy to show, that this last quantity has the expected size provided we can show for $1 \leq j \leq k$ that

$$
\sum_{q_{1}, q_{2} \in D} \sum_{\substack{n \in Z \\ q_{1}\left|n, q_{2}\right| n+j}} 1=\left(1+o_{k, \epsilon, \delta}(1)\right) \mathbb{E}(h)^{2}|Z| ;
$$

and, if we can do this, then if $k, \delta$ satisfy (8), we will have (9) for $x$ sufficiently large, thus proving our theorem.

In the sum on the left-hand-side of (12) we only need to consider $q_{1}, q_{2} \in D$ such that $\left(q_{1}, q_{2}\right)=1$, since we cannot have a prime $p>x^{12 / 95-\epsilon / 3}$ dividing both $n$ and $n+j$. Thus, to prove (12), we just need to count the number of integers $d$, and integers $q_{1}, q_{2} \in D,\left(q_{1}, q_{2}\right)=1$, satisfying the congruence

$$
d \equiv j q_{2}^{-1} \quad\left(\bmod q_{1}\right), \text { where } \frac{(1+\delta)^{-1} \sqrt{x}}{q_{2}}<d<\frac{(1+\delta) \sqrt{x}}{q_{2}}
$$

The fact that the range for $d$ depends on $q_{2}$ is problematic. We handle this by partitioning $D$ into $D_{1} \cup \cdots \cup D_{t}$, where $t=[\log x]$, and where $D_{i}$ is the set of all elements of $D$ lying in the interval

$$
\left[x^{24 / 95-2 \epsilon / 3}+(i-1) L_{0}, x^{24 / 95-2 \epsilon / 3}+i L_{0}\right) \text {, where } L_{0}=t^{-1} x^{24 / 95-\epsilon / 2},
$$

and by defining $I_{1}, \ldots, I_{t}$ to be integer intervals, where $I_{i}$ is the set of all integers $d$ satisfying

$$
\frac{(1+\delta)^{-1} \sqrt{x}}{\max \left\{q \in D_{i}\right\}} \leq d<\frac{(1+\delta) \sqrt{x}}{\min \left\{q \in D_{i}\right\}}
$$

Then, our number of solutions to (13) is

$$
\left(1+o_{k, \epsilon, \delta}(1)\right) \sum_{i=1}^{t} \sum_{q_{1} \in D} \sum_{\substack{q_{2} \in D_{i} \\\left(q_{2}, q_{1}\right)=1}} \sum_{\substack{d \in I_{i} \\ d \equiv j q_{2}^{-1}}} 1 .
$$

Good estimates for this quantity can be given through an application of exponential sums: First, define

$$
D_{i}(a, r)=\sum_{\substack{q \in D_{i} \\(q, r)=1}} e^{2 \pi i a[q] / r}, \text { where } q[q] \equiv 1 \quad(\bmod r)
$$


and define

$$
I_{i}(a, r)=\sum_{d \in I_{i}} e^{2 \pi i a d / r} .
$$

Then, our sums in (14) equal

$$
\sum_{i=1}^{t} \sum_{q_{1} \in D} \frac{1}{q_{1}} \sum_{|a|<q_{1} / 2} D_{i}\left(j a, q_{1}\right) I_{i}\left(-a, q_{1}\right) .
$$

The total contribution of the $a=0$ terms here is

$$
\left(1+o_{k, \delta, \epsilon}(1)\right) \sum_{i=1}^{t}\left|D_{i}\right|\left|I_{i}\right| \sum_{q_{1} \in D} \frac{1}{q_{1}}=\left(1+o_{k, \delta, \epsilon}(1)\right) \mathbb{E}(h) \sum_{i=1}^{t}\left|D_{i}\right|\left|I_{i}\right| .
$$

This last sum over $i$ counts, up to an error factor $1+o_{k, \delta, \epsilon}(1)$, the number of products $q_{2} d$ which lie in $Z$, where $q_{2} \in D$. Thus, the contribution of all the $a=0$ terms is

$$
\left(1+o_{k, \delta, \epsilon}(1)\right) \mathbb{E}(h)^{2}|Z|
$$

and so, proving (12) amounts to showing that the contribution of the terms $a \neq 0$ to $(15)$ is $o_{k, \delta, \epsilon}(|Z|)$.

\subsection{Kloosterman Sums}

To bound from above the terms in (15) where $a \neq 0$ we first fix $i=1, \ldots, t$, and $a$ satisfying

$$
a \neq 0,|a| \leq \max _{q_{1} \in D} q_{1} / 2<x^{24 / 95-\epsilon / 2} ;
$$

and then, we try to bound

$$
\sum_{q_{1} \in D} \frac{1}{q_{1}} D_{i}\left(j a, q_{1}\right) I_{i}\left(-a, q_{1}\right)
$$

from above in absolute value.

To carry out this plan, we will require the following very slight modification of a theorem of Duke, Friedlander, and Iwaniec [2, Theorem 2] on bilinear forms of Kloosterman sums: ${ }^{2}$

\footnotetext{
${ }^{2}$ The modification amounts to replacing the range on $n$ from $[N, 2 N]$ to $\left[N, N^{1+\epsilon_{0}}\right]$. This is easily handled by writing the sum with $\left[N, N^{1+\epsilon_{0}}\right]$ as a series of sums over dyadic intervals. Also, our version is stated for arbitrary $a$, whereas version in [2] is for $a \geq 0-$ the version for arbitrary $a$ is easily deduced by taking complex conjugates.
} 
Theorem 2 Suppose that $\left\{\alpha_{m}\right\}_{M<m \leq 2 M}$ and $\left\{\beta_{n}\right\}_{N<n \leq N^{1+\epsilon_{0}}}$ are sequences of complex numbers, and define

$$
\mathcal{B}(M, N, a)=\sum_{N<n \leq N^{1+\epsilon_{0}}} \beta_{n} \sum_{\substack{M<m \leq 2 M \\(m, n)=1}} \alpha_{m} e^{2 \pi i a[m] / n} .
$$

Then, for every $\epsilon_{1}>0$ and every integer a we have the following bound

$$
\mathcal{B}(M, N, a) \ll_{\epsilon_{1}} \quad\|\alpha\|_{2}|| \beta \|_{2}(|a|+M N)^{3 / 8}(M+N)^{11 / 48+\epsilon_{1}+2 \epsilon_{0} / 3}
$$

Here, $\|\alpha\|_{2}=\left(\sum_{i} \alpha_{i}^{2}\right)^{1 / 2}$.

Remark. In our case, the $\beta_{n}$ are supported on a set of integers $n=p_{1} p_{2}$, where $p_{i}<x^{12 / 95}$, and perhaps there is a way to take advantage of this special form to improve the bilinear form estimates in this case. If so, it would lead to an improvement to our main theorem.

We apply this theorem with

$$
M=N=\min _{q \in D} q=\left(1+o_{\epsilon}(1)\right) x^{24 / 95-2 \epsilon / 3}
$$

with

$$
\alpha_{m}= \begin{cases}1, & \text { if } m \in D_{i} \\ 0, & \text { if } m \notin D_{i}\end{cases}
$$

with

$$
\beta_{n}=\beta_{n}(j a)=\left\{\begin{aligned}
I_{i}(-a, n) / n, & \text { if } n \in D, \text { and } n>2|a| \\
0, & \text { otherwise }
\end{aligned}\right.
$$

and finally with $\epsilon_{0}=\epsilon / 6$ (we leave $\epsilon_{1}$ as a parameter that we choose as small as needed later). With this choice of parameters the expression in (16) equals $\mathcal{B}(M, N, j a)$, which for $|a|<\max _{q \in D} q / 2, a \neq 0$ is

$$
\left.\ll_{\epsilon_{1}}|| \beta(j a)\right|_{2}\left|D_{i}\right|^{1 / 2} x^{47 / 190+\epsilon_{1} / 3-3 \epsilon / 10}<\|\beta(j a)\|_{2} x^{71 / 190+\epsilon_{1} / 3-3 \epsilon / 4} .
$$

\subsection{Bounds on $\|\beta(a)\|_{2}$, and the Conclusion of the Proof}

In order to sum this over all these values of $a$, we require upper bounds on $\|\beta(j a)\|_{2}$ : We first realize that $I_{i}(-a, n)$ is a geometric series with common 
ratio $e^{-2 \pi i a / n}$; and so, using the notation $\|t\|$ for the distance from $t$ to the nearest integer, we get the bound

$$
\begin{aligned}
\left|I_{i}(-a, n)\right| & \leq \min \left(\left|I_{i}\right|, 2\left|e^{2 \pi i a / n}-1\right|^{-1}\right) \\
& =\min \left(\left|I_{i}\right|,(\sin \pi|| a / n||)^{-1}\right) \\
& \leq \min \left(\left|I_{i}\right|,(2|| a / n||)^{-1}\right) .
\end{aligned}
$$

This last inequality follows from the fact that $|\sin (t)| \geq 2|t| / \pi$.

Now, if $|a|<x^{1 / 190-\epsilon}$, then we will just use the bound $\left|I_{i}(-a, r)\right| \leq\left|I_{i}\right|$, which gives

$$
\|\beta(a)\|_{2}=\left|I_{i}\right|\left(\sum_{q_{1} \in D} \frac{1}{q_{1}^{2}}\right)^{1 / 2}<\left|I_{i}\right| x^{-12 / 95+\epsilon / 3}<x^{23 / 190+\epsilon} .
$$

On the other hand, when $|a|>x^{1 / 190-\epsilon}$ we use the bound $(2|| a / n||)^{-1}$, which gives

$$
\|\beta(j a)\|_{2} \leq\left(\sum_{\substack{q_{1} \in D \\ q_{1}>2|a|}} \frac{1}{4 q_{1}^{2}|| a /\left.q_{1}\right|^{2}}\right)^{1 / 2}<\frac{|D|^{1 / 2}}{2|a|}<\frac{x^{12 / 95-\epsilon / 4}}{|a|}
$$

Putting these bounds for $\|\beta(j a)\|_{2}$ together with the upper bound (17) for the expression (16), we find that

$$
\sum_{\substack{|a|<\max _{q \in D} \\ a \neq 0}} \sum_{\substack{q_{1} \in D \\ q_{1}>2|a|}} \frac{1}{q_{1}} D_{i}\left(j a, q_{1}\right) I_{i}\left(-a, q_{1}\right)=\Sigma_{1}+\Sigma_{2},
$$

where $\Sigma_{1}$ is the sum of terms where $a \neq 0$ satisfies $|a|<x^{1 / 190-\epsilon}$, and where $\Sigma_{2}$ is the sum of terms where $a \neq 0$ satisfies $|a|>x^{1 / 190-\epsilon}$. We have that

$$
\Sigma_{1} \ll\left(x^{1 / 190-\epsilon}\right) \max _{\substack{|a|<x^{1 / 190-3 \epsilon / 5} \\ a \neq 0}}\|\beta(j a)\|_{2} x^{71 / 190+\epsilon_{1} / 3-3 \epsilon / 4}<x^{1 / 2+\epsilon_{1} / 3-3 \epsilon / 4} ;
$$

likewise,

$$
\Sigma_{2} \ll x^{1 / 2+\epsilon_{1} / 3-\epsilon} \log x
$$

Thus, for $\epsilon_{1}=2 \epsilon$ we will get that the contribution of terms where $a \neq 0$ to (15) is $o_{k, \epsilon, \delta}\left(x^{1 / 2}\right)=o_{k, \epsilon, \delta}(|Z|)$, which is just what we needed to show in order to complete the proof of our theorem. 


\section{References}

[1] A. Balog, On the Distribution of Integers Having no Large prime Factor, Journées Arithmétiques, Besançon, Astérisque 147/148 (1985), 27-31.

[2] W. Duke, J. Friedlander, H. Iwaniec, Bilinear Forms with Kloosterman Fractions, Invent. Math. 128 (1997), 23-43.

[3] J. Friedlander and A. Granville, Smoothing "Smooth" Numbers, Philos. Trans. Roy. Soc. London Ser. A 345, 339-347.

[4] J. Friedlander and H. Iwaniec, The Brun-Titchmarsh Theorem, Analytic Number Theory (Kyoto 1996), 85-93.

[5] J. Friedlander and J. Lagarias, On the Distribution in Short Intervals of Integers Having No Large Prime Factor, J. Number Theory 25 (1987), 249-273.

[6] G. Harman, Integers without Large Prime Factors in Short Intervals and Arithmetic Progressions, Acta Arith. 91 (1999), 279-289.

[7] A. A. Karatsuba, Distribution of Inverse Values in a Residue Ring Modulo a Given Number, Dokl. Akad. Nauk 333 (1993), 452-454.

[8] H. W. Lenstra, Factoring Integers with Elliptic Curves, Ann. Math. 126 (1987), 649-673.

[9] T. Z. Xuan, On Smooth Integers in Short Intervals Under the Riemann Hypothesis, Acta Arith. 88, 327-332. 